\title{
Relating the QCD Pomeron to an effective gluon mass
}

\author{
F. Halzen \\ Deparment of Physics, University of Wisconsin, Madison, Wisconsin 53706 \\ G. Krein and A. A. Natale \\ Instituto de Física Teórica, Universidade Estadual Paulista, \\ Rua Pamplona 145, 01405, São Paulo, São Paulo, Brazil
}

(Received 29 May 1992)

\begin{abstract}
We construct the Pomeron as an exchange of two nonperturbative gluons, where the nonperturbative gluon propagator is described by an approximate solution of the Schwinger-Dyson equation which contains a dynamically generated gluon mass. We compute the total and elastic differential $(d \sigma / d t)$ cross sections for $p p$ scattering, obtaining agreement with the experimental data for a gluon mass $m=370 \mathrm{MeV}$ for $\Lambda_{\mathrm{QCD}}=300 \mathrm{MeV}$. In particular, the Pomeron effectively behaves like a photon-exchange diagram with a coupling determined by the gluon mass.

PACS number(s): 12.38. Lg, 12.38.Qk, 12.40.Gg, 13.85.Dz
\end{abstract}

It is known that Pomeron exchange gives a good description of the experimental data for total cross sections in hadron-hadron collisions, small- $|t|$ elastic scattering, and diffraction dissociation [1]. The phenomenological Pomeron exchange can be understood within QCD as the exchange of two gluons [2]; however, the perturbative calculation of the elastic quark-quark scattering amplitude through two-gluon exchange shows a singularity at $t=0$. Although this singularity can be canceled when incorporating quarks in the proton wave function, this procedure is not able to reproduce the $t$ dependence of the differential cross section observed in experiment [3]. The origin of this singularity is the pole in the gluon propagator at $k^{2}=0$, and it is clear that the small- $|t|$ behavior of the cross section cannot be explained in perturbative QCD. It was noticed by Landshoff and Nachtmann [4] that, if the gluon propagator is finite at $k^{2}=0$, the singularity in the two-gluon calculation of hadron-hadron scattering is eliminated. A behavior softer than a pole of the gluon propagator at small $k^{2}$ is expected from nonperturbative effects of the QCD vacuum which leads to confinement.

In this model the Pomeron is described by the exchange of two nonperturbative gluons, where by nonperturbative we mean a gluon whose propagator does not show a pole at $k^{2}=0$. Several phenomenological consequences of such an approach have already been discussed in the literature, assuming a simple form for the propagator [5]. Recently, Cudell and Ross [6] have solved an approximate Schwinger-Dyson (SD) equation for the gluon propagator in the axial gauge, and found a solution whose infrared behavior is less singular than a pole at $k^{2}=0$. This new solution for the gluon propagator, when incorporated in the Landshoff-Nachtmann model, gives reasonable agreement with the experimental data.

In the following we will show how a gluon propagator regularized by a dynamically generated gluon mass, derived by Cornwall some years ago [7], successfully describes nucleon-nucleon scattering in the two-gluonexchange model. The propagator represents an approximate solution of the Schwinger-Dyson equation and, unlike the propagator obtained by Cudell and Ross, it asymptotically obeys the renormalization group equation and is actually finite at $k^{2}=0$ as a result of the effective gluon mass. The model is described by a single parameter which is taken to be the ratio of the gluon mass and $\Lambda=\Lambda_{\mathrm{QCD}}$.

In the Landshoff-Nachtmann model Pomeron exchange between quarks behaves like a photon-exchange diagram with an amplitude

$$
i \beta_{0}^{2}\left(\bar{u} \gamma_{\mu} u\right)\left(\bar{u} \gamma^{\mu} u\right)
$$

where $\beta_{0}$ is the strength of the Pomeron coupling to quarks which is given by

$$
\beta_{0}^{2}=\frac{1}{36 \pi^{2}} \int d^{2} k\left[g^{2} D\left(k^{2}\right)\right]^{2} .
$$

The origin is familiar from QED: multiple-photon exchanges only modify the single-exchange amplitude by a Coulomb phase. The advantage of using an infraredfinite propagator is that the integral in Eq. (2) converges. Particularly, this solves the problems with factorization discussed in detail by Landshoff and Nachtmann [4]. This is not the case for most of the Schwinger-Dyson solutions for the gluon propagator found in the literature [6, 11]. We will show that in our model the phenomenological value $\beta_{0}$ of $2 \mathrm{GeV}^{-1}$ [1], determined from the total cross section, is accommodated by a gluon mass $m \simeq 2(1.2) \Lambda_{\mathrm{QCD}}$ for $\Lambda_{\mathrm{QCD}}=150(300) \mathrm{MeV}$. We will show that these parameters also describe the experimental features of forward proton-proton scattering.

The possibility that the gluon has a dynamically generated mass has been advocated by Cornwall in a series of papers $[7,8]$. Lattice calculations also indicate that the gluon behaves like a massive particle [9]. A bare gluon mass has attractive phenomenological implications [10], although it leads to known problems with unitarity. This problem is avoided in the case of a dynamical mass. Approximate solutions of the SchwingerDyson equation, obtained in a particular gauge, do not 
result in a dynamical gluon mass generation $[6,11]$. Cornwall has, however, identified a gauge-invariant set of diagrams for the Schwinger-Dyson equation which allows for a finite (and different from zero) result at $k^{2}=0$. Most importantly, he also found a massive trial propagator that gives an excellent fit to the numerical solution. This solution incorporates the correct ultraviolet behavior of the gluon propagator according to the renormalization group. Because of ambiguities in numerical solutions of the Schwinger-Dyson equation it is much more important to have an approximate solution which obeys the renormalization group than a single numerical result where such behavior is not directly observed [7].

In the Feynman gauge the propagator is given by $D_{\mu \nu}=-i g_{\mu \nu} D\left(q^{2}\right)$, where (in Euclidean space) [7]

$D^{-1}\left(q^{2}\right)=\left[q^{2}+m^{2}\left(q^{2}\right)\right] b g^{2} \ln \left[\frac{q^{2}+4 m^{2}\left(q^{2}\right)}{\Lambda^{2}}\right]$,

with the momentum-dependent dynamical mass given by

$$
m^{2}\left(q^{2}\right)=m^{2}\left[\frac{\ln \left(\frac{q^{2}+4 m^{2}}{\Lambda^{2}}\right)}{\ln \frac{4 m^{2}}{\Lambda^{2}}}\right]^{-12 / 11} .
$$

In the above equations $m$ is the gluon mass, and $b=$ $\left(33-2 n_{f}\right) / 48 \pi^{2}$ is the leading-order coefficient of the $\beta$ function of the renormalization group equation. The coupling $g$ is frozen and $g^{2} D\left(q^{2}\right)$ is formally independent of $g$. The solution is valid only for $m>\Lambda / 2$ [7]. Relating the gluon mass to the gluon condensate, Cornwall estimated $m=500 \pm 200 \mathrm{MeV}$ for $\Lambda_{\mathrm{QCD}}=300 \mathrm{MeV}$.

The description of the Pomeron by the exchange of two nonperturbative gluons is necessarily crude, e.g., the calculation does not include multiple-gluon effects described by the Lipatov equation [12]. The model does not incorporate an intercept larger than 1 and therefore cross sections are independent of energy. In QCDinspired models [13] of the total cross section our model of the Pomeron would describe the energy-independent part of the cross section associated with the interactions of the valence quarks. The rise of the cross section with energy is associated with the increasing number of gluons in very-high-energy hadrons. Using a Regge parametrization the total $p p$ cross section is fitted by $\sigma_{T}(p p) \simeq 22.7\left(s / m_{p}^{2}\right)^{0.08}$, where $m_{p}$ is the proton mass [1], and the "bare" Pomeron thus accounts for $22.7 \mathrm{mb}$ energy-independent cross section. The model should also reproduce the differential cross section for $p p$ elastic scattering up to $-t \simeq 0.5 \mathrm{GeV}^{2}$, for larger $|t|$ values the cross section will not be purely imaginary and will receive important contributions from multiple Pomeron exchange [1].

Notice that in QCD-inspired models the energy independent part of the total cross section is roughly $30 \mathrm{mb}$ [13]. The variation from model to model for the value of the constant part of the total cross section is just a reflection of the different fits to the energy dependence exhibited by the data. We will use the previously quoted value of $22.7 \mathrm{mb}$ as representative.

The amplitude for elastic proton-proton scattering via two-gluon exchange can be written as [14]

$$
A(s, t)=i s 8 \alpha_{s}^{2}\left[T_{1}-T_{2}\right],
$$

with

$$
\begin{aligned}
T_{1}=\int_{0}^{s} d^{2} k D\left(\frac{q}{2}+k\right) D\left(\frac{q}{2}-k\right)\left[G_{p}(q, 0)\right]^{2} \\
T_{2}=\int_{0}^{s} d^{2} k D\left(\frac{q}{2}+k\right) D\left(\frac{q}{2}-k\right) G_{p}\left(q, k-\frac{q}{2}\right) \\
\quad \times\left[2 G_{p}(q, 0)-G_{p}\left(q, k-\frac{q}{2}\right)\right],
\end{aligned}
$$

where $\alpha_{s}=g^{2} / 4 \pi$ is the strong coupling constant, $D(k)$ the gluon propagator given by Eq. (3), and $G_{p}(q, k)$ a convolution of proton wave functions:

$G_{p}(q, k)=\int d^{2} p d \alpha \psi^{*}(\alpha, p) \psi(\alpha, p-k-\alpha q)$.

The wave function $\psi(\alpha, p)$ describes the amplitude for a quark of transverse momentum $p$ and fractional longitudinal momentum $\alpha . T_{1}$ represents contributions from diagrams where both gluons are attached to the same quark within the proton, whereas $T_{2}$ comes from diagrams in which the gluons are attached to different quarks. $G_{p}(q, 0)$ is given by the Dirac form factor of the proton

$$
F_{1}(t)=\frac{4 m^{2}-2.79 t}{4 m^{2}-t} \frac{1}{(1-t / 0.71)^{2}} .
$$

To estimate $G_{p}(q, k-q / 2)$ we assume a proton wave function peaked at $\alpha=1 / 3$ and use [6] $G_{p}(q, k-q / 2)=$ $F_{1}\left(q^{2}+9\left|k^{2}-q^{2} / 4\right|\right)$.

From the amplitude of Eq. (5) we obtain both the total cross section $\sigma_{T}=A(s, 0) / i s$ and the differential cross section $d \sigma / d t=|A(s, t)|^{2} /\left(16 \pi s^{2}\right)$. For very small $|t|$ values the elastic differential cross section can be fitted as $d \sigma / d t=A e^{B t}$, where $B$ is the logarithmic slope of the forward amplitude. The validity of the model requires that a reasonable value of the gluon mass $m$ yields $\sigma_{0}=$ $22.7 \mathrm{mb} . B$ and $d \sigma / d t$ are subsequently predicted. We will make this comparison for a c.m. energy of $\sqrt{s}=$ $53 \mathrm{GeV}$, i.e., an energy where the rising component of the total cross section does not significantly contribute. At this energy $B=11.06 \pm 0.11 \mathrm{GeV}^{-2}$ and $(d \sigma / d t)_{t=0}=$ $94.0 \pm 1.0 \mathrm{mb} / \mathrm{GeV}^{2}[15]$.

In Fig. 1 we show the total cross section for $p p$ scattering as a function of the gluon mass. The different curves are for different values of $\Lambda$. Although the model is basically parametrized in terms of their ratio, there is some residual dependence on the gluon mass and $\Lambda$. Notice that for increasing $\Lambda$ agreement with $\sigma_{T}$ is only obtained by decreasing the ratio $m / \Lambda$. Increasing $\Lambda$ only yields the proper value of $\sigma_{T}$ for smaller $m$, and here one must remember that Eq. (3) only solves the SchwingerDyson equation for $m>\Lambda / 2$ [7]. Only a small region in $(m, \Lambda)$ parameter space remains when fitting the full set of data $\left(\sigma_{T}\right.$ or $\beta_{0}$ and $\left.B\right)$. We obtain excellent agreement 


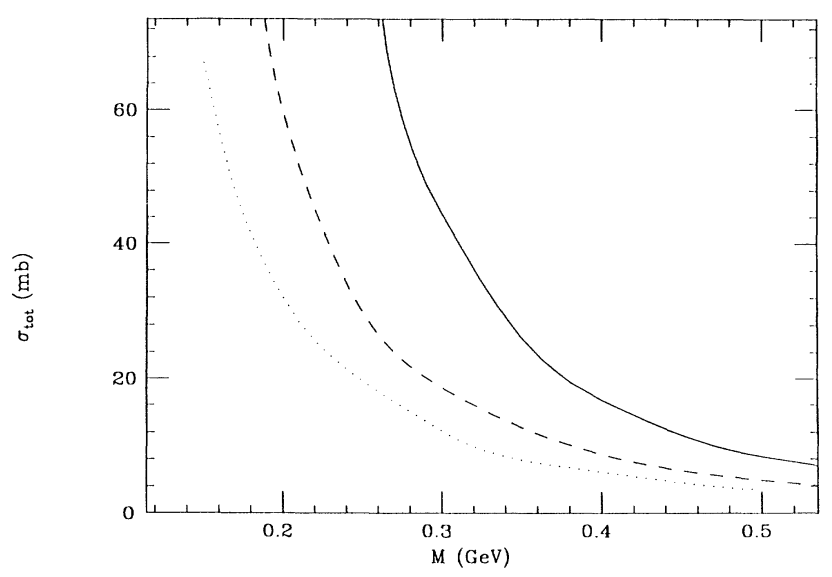

FIG. 1. Total cross section $\left(\sigma_{T}\right)$ as a function of the gluon mass $m$ for different values of $\Lambda: \Lambda=300 \mathrm{MeV}$ (solid curve), $\Lambda=200 \mathrm{MeV}$ (dashed curve), and $\Lambda=150 \mathrm{MeV}$ (dotted curve).

between theory and experiment for $m=370 \mathrm{MeV}$ and $\Lambda=300 \mathrm{MeV}$. These parameters yield $\sigma_{0}=21.6 \mathrm{mb}$, $B=11.5 \mathrm{GeV}^{-2}$, and $\beta_{0}=2.3 \mathrm{GeV}^{-1}$.

In Fig. 2 we show the slope $B$ for small $|t|$ as a function of $\Lambda$. The curves represent different values of the ratio $m / \Lambda$. For $\Lambda$ in the interval of 150 to $300 \mathrm{GeV}$, we verify that smaller $\Lambda$ values require larger masses.

That this simple model nicely accommodates $d \sigma / d t$ can be seen in Fig. 3. Our results have to be adjusted by a normalization factor $s^{0.168}$ which accounts for the energydependent part of $d \sigma / d t$ [1]. The excellent fit implies that the energy dependent part cannot have a drastically different structure in impact parameter. This is true in most models, see, e.g., Ref. [13]. For larger $|t|$ values, double-Pomeron exchange and three-gluon exchange are known to be important; we therefore do not expect to describe the data near and above $-t \simeq 0.5 \mathrm{GeV}^{2}[1,16]$.

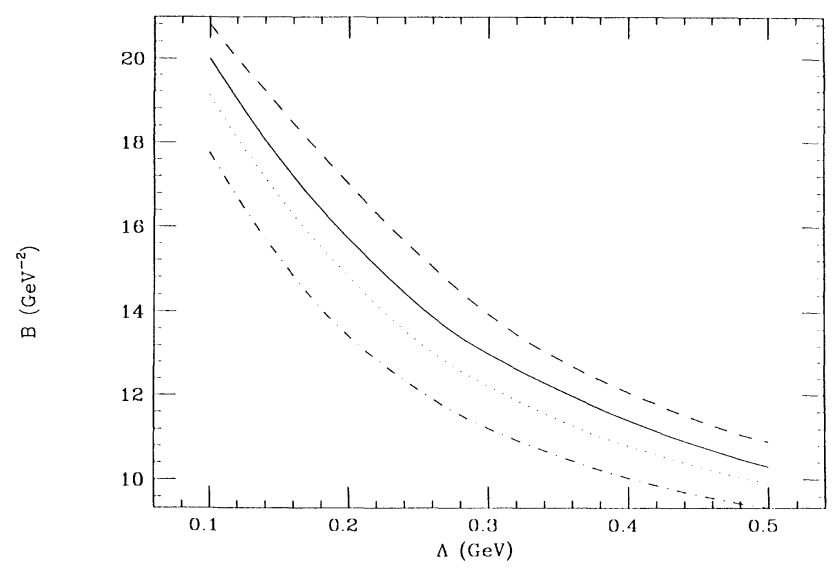

FIG. 2. Slope of the differential $p p$ scattering cross section as a function of $\Lambda$. The different curves represent different values of the ratio $m / \Lambda: m / \Lambda=0.9$ (dashed curve), $m / \Lambda=1$ (solid curve), $m / \Lambda=1.1$ (dotted curve), and $m / \Lambda=1.3$ (dotdashed curve).

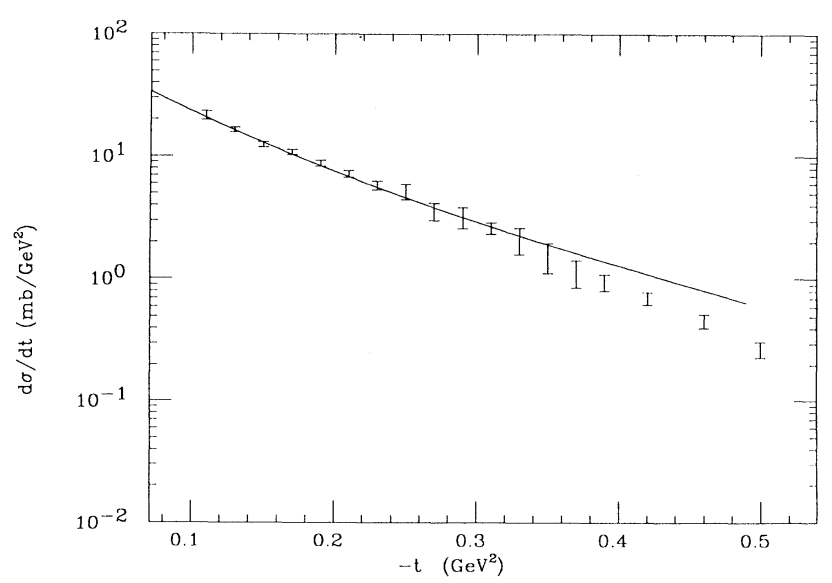

FIG. 3. Differential $p p$ elastic cross section obtained from double nonperturbative gluon exchange, with $m=370 \mathrm{MeV}$ and $\Lambda=300 \mathrm{MeV}$ (solid curve). Data from Ref. [15] at $\sqrt{s}=$ $53 \mathrm{GeV}$.

The above fits assumed a nominal value of $22.7 \mathrm{mb}$ for the energy-independent part of the total cross section. Different models for the energy dependence of the total cross section lead to a range of values between 20 and $30 \mathrm{mb}$. This range corresponds to a spread in the gluon mass of $m \simeq 2(1.2) \Lambda_{\mathrm{QCD}}$ for $\Lambda_{\mathrm{QCD}}=150$ (300) $\mathrm{MeV}$.

In Eq. (5) the amplitude $T_{1}$ where the gluons couple to the same quark recovers the factorizable, additive quark model, $T_{2}$ does not. For the fitted values of the gluon mass $T_{1}$ is slightly larger than $T_{2}$ with their ratio increasing for larger gluon masses. The fact that $T_{1}$ is larger than $T_{2}$ implies that the additive quark model is valid in an approximate sense. In our model this result is not critically dependent on the form of hadronic wave functions.

For $|t|$ of the order of $3 \mathrm{GeV}^{2}$ or larger, triple-gluon exchange is expected to dominate the $p p$ scattering cross section, and its contribution has the form [16]

$$
\frac{d \sigma}{d t} \simeq C t^{-8}\left[\alpha_{s}(t)\right]^{6} .
$$

The experimental data can be fitted by a $t^{-8}$ behavior with a coefficient $C$ which is approximately independent of energy. However, if $\alpha_{s}$ in Eq. (10) is allowed to run agreement between the theory and experiment is ruined. Notice however, that if the exchange of the three gluons is described by the propagator of Eq. (3) which is of the $O\left(1 / \alpha_{s}^{2}\right)$, the coupling constants in Eq. (10) are exactly canceled.

In conclusion, we verified that a model where the Pomeron is described by the exchange of two nonperturbative gluons with massive propagators provides an excellent description of small-angle $p p$ scattering. Even for moderate values of $|t|$, where triple gluon exchange dominates the behavior of the differential cross section, the propagator given by Eq. (3) may give a hint of how an apparent contradiction between theory and experiment [16] can be resolved. Finally, the determination of a dynamical gluon mass of order $1.2 \sim 2 \Lambda_{\mathrm{QCD}}$ is consis- 
tent with totally unrelated estimates [7]. If we assume an energy-independent part of the total cross section of $22.7 \mathrm{mb}$, then a best fit to the experimental data is obtained for $m=370 \mathrm{MeV}$ when $\Lambda_{\mathrm{QCD}}=300 \mathrm{MeV}$. Since a massive gluon propagator has many other phenomenological implications $[5,10]$, a study of these together with the total cross section calculation, may provide a tighter relation between $m$ and $\Lambda_{\mathrm{QCD}}$.

We thank Jean-Rene Cudell and Peter Landshoff for many constructive comments on an early version of this paper. This research was supported in part by the University of Wisconsin Research Committee with funds granted by the Wisconsin Alumni Research Foundation, by the U.S. Department of Energy under Contract No. DE-AC02-76ER00881, by the Texas National Research Laboratory Commission under Grant No. RGFY9173, and by the Conselho Nacional de Desenvolvimento Cientifico e Tecnologico (CNPq).
[1] P. V. Landshoff, in Proceedings of the Joint International Lepton-Photon Symposium and Europhysics Conference on High Energy Physics, Geneva, Switzerland, 1991, edited by S. Hegarty, K. Potter, and E. Quercigh (World Scientific, Singapore, 1992); Report No. CERNTH-6277/91 (unpublished).

[2] F. E. Low, Phys. Rev. D 12, 163 (1975); S. Nussinov, Phys. Rev. Lett. 34, 1268 (1975).

[3] D. G. Richards, Nucl. Phys. B258, 267 (1985).

[4] P. V. Landshoff and O. Nachtmann, Z. Phys. C 35, 405 (1987).

[5] A. Donnachie and P. V. Landshoff, Nucl. Phys. B311, $509(1988 / 89)$; J. R. Cudell, A. Donnachie, and P. V. Landshoff, ibid. B322, 55 (1989); J. R. Cudell, ibid. B336, 1 (1990).

[6] J. R. Cudell and D. A. Ross, Nucl. Phys. B359, 247 (1991).

[7] J. M. Cornwall, Phys. Rev. D 26, 1453 (1982).

[8] J. M. Cornwall, in Deeper Pathways in High-Energy Physics, edited by B. Kursunoglu, A. Perlmutter, and L. Scott (Plenum, New York, 1977), p. 683; Nucl. Phys. B157, 392 (1979).
[9] C. Bernard, Phys. Lett. 108B, 431 (1982); P. A. Amundsen and J. Greensite, Phys. Lett. B 173, 179 (1986); J. E. Mandula and M. Ogilvie, ibid. 185, 127 (1987).

[10] G. Parisi and R. Petronzio, Phys. Lett. 94B, 51 (1980).

[11] M. Baker, J. S. Ball, and F. Zachariasen, Nucl. Phys. B186, 531 (1981); B186, 560 (1981); N. Brown and M. R. Pennington, Phys. Rev. D 38, 2266 (1988); 39, 2723 (1989).

[12] L. N. Lipatov, and G. V. Frolov, Yad. Fiz. 13, 588 (1971) [Sov. J. Nucl. Phys. 13, 333 (1971)]; E. Kuraev, L. Lipatov, and V. Fadin, Zh. Eksp. Teor. Fiz. 72, 377 (1977) [Sov. Phys. JETP 45, 199 (1977)]; Y. Balitsky and L. Lipatov, Yad. Fiz. 28, 1597 (1978) [Sov. J. Nucl. Phys. 28, 822 (1978)].

[13] M. M. Block, F. Halzen, and B. Margolis, Phys. Rev. D 45, 839 (1992), and references therein.

[14] J. F. Gunion and D. Soper, Phys. Rev. D 15, 2617 (1977); E. M. Levin and M. G. Ryskin, Yad. Fiz. 41, 1622 (1985) [Sov. J. Nucl. Phys. 41, 1027 (1985)].

[15] A. Breakstone et al., Nucl. Phys. B248, 253 (1984).

[16] A. Donnachie and P. V. Landshoff, Nucl. Phys. B231, 189 (1984). 